\title{
Motivação para a prática de balé clássico por mulheres adultas
}

\section{Motivation for classic ballet practice by adult women}

\section{AUTORES \\ Viviane Maria Moraes de Oliveira ${ }^{1}$ Juliana Siqueira Lopes² (D) \\ 1 Associação Caruaruense de Ensino Superior, Departamento de Educação Física. Caruaru, Pernambuco, Brasil. \\ 2 Centro Universitário FBV Wyden, UniFBV Wyden, Departamento de Educação Física. Recife, Pernambuco, Brasil.}

\section{CONTATO}

Viviane Maria Moraes de Oliveira vivimmo@hotmail.com

Rua Conde Pereira Carneiro, 305, apt 704B, Imbiribeira, Recife, Pernambuco, Brasil. CEP: 51150-310.

DOI

$10.12820 /$ rbafs. $25 \mathrm{e} 0129$

\section{(cc) BY}

Este trabalho está licenciado com uma Licença Creative Commons - Atribuição 4.0 Internacional.

\begin{abstract}
RESUMO
A motivação para a prática de qualquer exercício físico é a base determinante para conhecer a escolha da modalidade, adesão e a desistência da mesma. O objetivo deste estudo foi identificar os fatores que levam a mulher adulta a escolher o balé clássico enquanto atividade física, bem como verificar se existe associação significativa entre as dimensões motivacionais para a prática do balé segundo o tempo de prática e grupo de idades. Foi aplicado o questionário Inventário de Motivação à Prática Regular de Atividade Física em 42 mulheres que iniciaram a prática do balé clássico após os 18 anos de idade, e que praticam a modalidade em escolas de danças na cidade de Caruaru, Pernambuco. As mulheres optaram pelo balé clssico por diversos motivos, sendo os principais deles prazer e sociabilidade. Além disso, a competitividade foi um fator motivacional que se destacou entre as participantes mais jovens .
\end{abstract}

Palavras-chave: Motivação; Dança; Atividade física.

\begin{abstract}
The motivation to practice any exercise is the determining basis for knowing the choice of the modality, adherence and the withdrawal from it. The aim of this study was to identify the factors that lead adult women to choose classical ballet as a physical activity. As well as checking if there is a significant association between the motivational dimensions for the practice of ballet according to the time of practice and age group. The Motivation Inventory Questionnaire for Regular Physical Activity was applied to 42 women who started classical ballet after 18 years of age, and who practice the modality in dance schools in the city of Caruaru, Pernambuco. Women opted for classical ballet for several reasons, the main ones being pleasure and sociability, in addition competitiveness was a motivational factor that stood out among the younger participants.
\end{abstract}

Keywords: Motivation; Dancing; Physical activity.

\section{Introdução}

A motivação é a força que emerge, regula e sustenta as ações de cada indivíduo; ela é um processo complexo que influencia o início de uma atividade e a sua manutenção com persistência e vigor ao longo do tempo ${ }^{1}$. Quando se refere à execução de uma determinada atividade pelo prazer que esta pode proporcionar, é chamada motivação intrínseca. Já a motivação extrínseca apresenta-se como a motivação para trabalhar em resposta a algo externo à tarefa ou atividade, como para a obtenção de recompensas materiais ou sociais de reconhecimento ${ }^{2}$.

Conhecer os motivos que determinam a prática de uma atividade física tem sido um tema de frequente investigação na área da atividade física e saúde ${ }^{3,4}$. De um modo geral, os estudos destacam que a motivação para a prática de qualquer exercício é a base determinante para conhecer a escolha da modalidade, adesão e a desistência da mesma ${ }^{3,5}$ A falta de motivação às práticas saudáveis ou a escolha inadequada da atividade em relação ao perfil do indivíduo cria um alto índice de desistência da prática ${ }^{6}$.

No que diz respeito à prática de atividade física por mulheres adultas, Marbá et al. ${ }^{7}$, apontam como principais fatores motivacionais valores como o desafio, a visão corporal, o bem-estar, a qualidade de vida, a estética e a relação social. Além disso, as mulheres tendem a buscar a atividade física devido à preocupação com sua imagem corporal ${ }^{8}$. Dentre diversas atividades, Balbinotti \& Capozzoli ${ }^{9}$ apontam a dança como uma prática comum entre as mulheres, e embora esta possa ser compreendida como uma atividade física, o componente artístico diferencia a dança da maioria dos esportes ${ }^{10}$.

Observa-se que no balé clássico, arte de tradição secular, cujas origens remontam a mais de 500 anos de evolução técnica e estética ${ }^{11}$, o início da prática cos- 
tuma acontecer ainda na infância, possivelmente pelo interesse de alguns jovens praticantes em uma possível carreira profissional ${ }^{12}$. Para além disso, um programa de formação em balé clássico requer anos de prática, devido ao seu extenso conteúdo. "Num curso regular de formação de bailarinos clássicos o programa abrange oito ou nove anos de estudo e é construído para contemplar estudantes entre oito e dezoito anos"13, sem contar com as aulas anteriores à formação técnica propriamente dita, também conhecidas como baby class ou pré-balé, quando se trabalham os rudimentos do balé clássico, ou ainda aulas de dança criativa, em que os aprendizes entram em contato com uma atividade rítmica sem necessariamente ter como referência o balé clássico; o início dessas atividades pode variar, mas geralmente são aceitas crianças de três anos de idade para a dança criativa e cinco anos para o baby class $^{14}$.

Assim, tradicionalmente a dança clássica tem sido procurada pelas primeiras idades, ou por pessoas que praticam esta forma de dança há bastante tempo, mesmo atualmente, em que muitos não buscam a profissionalização no balé ${ }^{12}$. Os pais sentem-se motivados a matricular seus filhos, especialmente meninas, desde muito cedo em classes de balé ${ }^{15}$. Nesse contexto, na idade adulta o praticante de balé já acumula anos de experiência podendo vir ou não a alcançar uma carreira profissional ${ }^{16}$.

Porém, o que se observa atualmente é que a aderência por mulheres que iniciam a prática no balé clássico após atingir a idade adulta tem sido cada vez maior, e muitas vezes esta procura dialoga com a vontade de ter uma prática corporal associada à arte, inspirando-se não somente pelo movimento, mas por valores ligados ao imaginário do balé clássico, que nos dias atuais está muito relacionado à figura feminina ${ }^{12}$. Normalmente, as mulheres praticantes inserem-se em duas realidades: ou tiveram a oportunidade de dançar balé apenas após a idade adulta, ou são ex-praticantes que dançaram quando crianças e desejam repetir a experiência ${ }^{16}$.

Embora seja observado que a prática do balé clássico por mulheres adultas venha se propagando de maneira intensa nas escolas de dança, não é comum encontrar estudos que relacionem os fatores motivacionais para a adesão e permanência dessa população nessa atividade. Iniciar a prática no balé clássico na idade adulta é um fenômeno relativamente recente, e, portanto, necessita de maior compreensão em diferentes perspectivas. $\mathrm{Co}^{-}$ nhecer os motivos que impulsionam mulheres adultas a aderirem à prática do balé clássico pode contribuir de maneira significativa no planejamento pedagógico da prática, de modo a garantir também uma maior permanência por parte destas na atividade.

Partindo do princípio que para programar intervenções que sejam eficazes no campo da atividade física é necessário conhecer a motivação do grupo e os fatores associados à prática de atividade física no ambiente estudado ${ }^{17}$, o presente estudo teve como objetivo identificar os fatores que levam a mulher adulta a escolher o balé clássico enquanto atividade física, buscando verificar, ainda, se existe associação significativa entre as dimensões motivacionais para a prática do balé segundo o tempo de prática e a idade.

\section{Métodos}

O estudo caracterizou-se como uma pesquisa de abordagem quantitativa de delineamento descritivo. A amostra por conveniência foi composta por 42 mulheres devidamente matriculadas em estúdios ou escolas de dança da cidade de Caruaru, Pernambuco, que começaram a praticar o balé clássico após os 18 anos de idade. Após serem convidadas para integrar a pesquisa, todas as mulheres que se encontravam presentes aceitaram fazer parte e assinaram Termo de Consentimento Livre e Esclarecido, conforme a resolução 466/12 da Comissão Nacional de Ética em Pesquisa do Ministério da Saúde do Brasil. Dessa forma, não houve perdas e recusas durante a pesquisa.

A pesquisa foi aprovada pelo Comitê de Ética do Centro Universitário Tabosa de Almeida ASCES-UNITA (CAEE: 80391317.6.0000.5203).

Foi aplicado o questionário Inventário de Motivação à Prática Regular de Atividade Física (IMPRAF - 126), desenvolvido por Balbitoni ${ }^{18}$, que tem como objetivo avaliar seis das possíveis dimensões associadas à motivação para a prática de atividades físicas de maneira regular. Através de 120 itens agrupados seis a seis, pode-se observar uma sequência: primeiro bloco de seis, apresenta questões relativas à dimensão motivacional, Controle de Estresse (CE), o segundo se trata de Saúde (SA) a terceira Sociabilidade (SO), a quinta relata sobre Estética (ES) e por último, a sexta aborda aspectos sobre Prazer (PR). Esse modelo foi repetido no segundo bloco que possui seis questões, até completar vinte blocos, e o bloco 21 é composto por seis questões repetidas. Seu objetivo é verificar um grau de concordância acordada a primeira e a segunda resposta ao mesmo item. As respostas do inventário foram dadas conforme a escala bidirecional de tipo Likert, graduada em 5 pontos, possibilitando as respostas: "isso me mo- 
tiva pouquíssimo" (1); "isso me motiva pouco"(2); "mais ou menos - não sei dizer - tenho dúvida" (3); "isso me motiva muito" (4); "isso me motiva muitíssimo" (5).

As participantes também preencheram um formulário contendo informações como idade e tempo de prática do balé em anos. Para a análise dos dados, as idades foram agrupadas da seguinte forma: de 18 a 20 anos (grupo 1), de 21 a 30 anos (grupo 2), de 31 anos ou mais (grupo 3).

O tempo de prática do balé foi considerado como até um ano de prática e acima de um ano de prática. Embora a maioria dos estudos referentes à motivação para prática de atividades físicas geralmente estabeleça o tempo de seis meses como viável para classificar o indivíduo enquanto adepto à prática ${ }^{19,20}$, em nossa amostra não houveram participantes com um tempo menor do que seis meses, dessa forma classificamos a amostra considerando o período de um ano de prática da atividade. $\mathrm{O}$ questionário e formulário foram aplicados no período de dois meses, entre fevereiro e março de 2017.

Os dados foram analisados descritiva e inferencialmente. A análise descritiva foi através de distribuições absolutas, percentuais para variáveis categóricas e as medidas estatísticas: média, desvio padrão, mediana e os percentis 25 e 75 para as variáveis numéricas e a análise inferencial através dos testes estatísticos. Para a comparação das variáveis numéricas entre as duas categorias foi utilizado o t-Student com variâncias iguais, t-Student com variâncias desiguais ou o teste de Mann-Whitney; na comparação de três categorias foi utilizado o teste F (ANOVA) ou Kruskal-Wallis.

Os testes t-Student e F (ANOVA) foram utilizados nas situações em que foi verificada a hipótese de normalidade dos dados e os testes de Mann-Whitney e Kruskal-Wallis nos casos da rejeição da normalidade em pelo menos uma das categorias. A verificação da normalidade dos dados foi realizada através do teste de Shapiro-Wilk e a igualdade de variâncias através do teste $\mathrm{F}$ de Levene.

A margem de erro utilizada nas decisões dos testes estatísticos foi de 5\%. Os dados foram digitados na planilha Excel e programa estatístico utilizado para obtenção dos cálculos estatísticos foi o IMB SPSS na versão 23.

\section{Resultados}

A Tabela 1 apresenta os resultados relativos às características da amostra. Ressalta que a faixa etária 21 a 30 anos foi a mais prevalente com $42,9 \%$ da amostra. Um pouco mais da metade $(57,1 \%)$ das participantes apresentou tempo de prática até um ano e $28,6 \%$ das participantes afirmou praticar outras atividades físicas.
A Tabela 2 apresenta as estatísticas das dimensões motivacionais, onde é possível verificar que a média mais elevada correspondeu à dimensão prazer (43,33 \pm $2,56)$, seguido da sociabilidade $(38,24 \pm 5,71)$. A competitividade não foi uma dimensão motivacional que se destacou entre os resultados $(11,69 \pm 4,92)$.

A Tabela 3 apresenta as estatísticas das dimensões motivacionais segundo o tempo de prática do balé e a Tabela 4 segundo grupo de idades das pesquisadas.

Tabela 1 - Características da amostra no grupo total $(n=42)$

\begin{tabular}{lcc}
\hline \multicolumn{1}{c}{ Variável } & $\mathrm{n}$ & $\%$ \\
\hline Grupo de idades (anos) & 8 & 19,0 \\
18 a 20 (grupo 1) & 18 & 42,9 \\
21 a 30 (grupo 2) & 16 & 38,1 \\
31 ou mais (grupo 3) & & \\
Tempo que pratica balé & 24 & 57,1 \\
Até um ano & 18 & 42,9 \\
Acima de um ano & & \\
Prática de outras atividades físicas & 12 & 28,6 \\
Sim & 30 & 71,4 \\
Não &
\end{tabular}

Tabela 2 - Estatísticas das dimensões motivacionais

\begin{tabular}{ll}
\hline Dimensão & Média \pm DP \\
\hline Prazer & $43,33 \pm 2,56$ \\
Sociabilidade & $38,24 \pm 5,71$ \\
Estética & $37,17 \pm 5,57$ \\
Saúde & $33,14 \pm 7,55$ \\
Controle de stress & $27,36 \pm 8,18$ \\
Competitividade & $11,69 \pm 4,92$ \\
\hline
\end{tabular}

Tabela 3 - Estatísticas das dimensões motivacionais segundo o tempo de prática

\begin{tabular}{|c|c|c|c|}
\hline \multirow{4}{*}{ Dimensão } & \multicolumn{2}{|c|}{ Tempo de prática do balé } & \multirow{4}{*}{ Valor $\mathrm{p}$} \\
\hline & Até 1 ano & Acima de 1 ano & \\
\hline & Média \pm DP & Média \pm DP & \\
\hline & Mediana (P25; P75) & Mediana (P25; P75) & \\
\hline \multirow{2}{*}{ Controle de stress } & $27,58 \pm 7,37$ & $27,06 \pm 9,37$ & \multirow[t]{2}{*}{$0,796^{2}$} \\
\hline & $28,50(21,25 ; 31,75)$ & $30,00(18,75 ; 35,00)$ & \\
\hline \multirow{2}{*}{ Saúde } & $33,71 \pm 8,57$ & $32,39 \pm 6,09$ & \multirow[t]{2}{*}{$0,582^{b}$} \\
\hline & $36,00(27,50 ; 40,00)$ & $31,00(28,75 ; 36,50)$ & \\
\hline \multirow{2}{*}{ Sociabilidade } & $38,25 \pm 6,69$ & $38,22 \pm 4,25$ & \multirow[t]{2}{*}{$0,564^{a}$} \\
\hline & $39,50(34,25 ; 44,75)$ & $39,00(34,75 ; 40,50)$ & \\
\hline \multirow{2}{*}{ Competitividade } & $12,04 \pm 5,47$ & $11,22 \pm 4,17$ & \multirow[t]{2}{*}{$0,893^{a}$} \\
\hline & $9,00(9,00 ; 14,25)$ & $9,00(9,00 ; 10,50)$ & \\
\hline \multirow{2}{*}{ Estética } & $36,08 \pm 6,29$ & $38,61 \pm 4,16$ & \multirow[t]{2}{*}{$0,126^{\circ}$} \\
\hline & $37,00(30,25 ; 40,00)$ & $40,00(35,75 ; 40,00)$ & \\
\hline \multirow{2}{*}{ Prazer } & $42,92 \pm 2,57$ & $43,89 \pm 2,52$ & \multirow[t]{2}{*}{$0,16^{\mathrm{a}}$} \\
\hline & $45,00(40,00 ; 45,00)$ & $45,00(43,75 ; 45,00)$ & \\
\hline
\end{tabular}

$\mathrm{a}=$ Teste Mann-Whitney; $\mathrm{b}=\mathrm{t}-$ Student com variâncias iguais; $\mathrm{c}=$ $\mathrm{t}$-Student com variâncias desiguais. 
Não foram registradas diferenças significativas entre as duas categorias do tempo de prática para a margem de erro fixada (5\%) em nenhuma dimensão.

Dos resultados contidos na Tabela 4 verifica-se que "Competitividade" foi a única diferença significativa ( $\mathrm{p}$ $<0,05)$ entre as faixas etárias e para a referida variável salienta-se que a média foi mais elevada na faixa etária de 18 a 20 anos $(16,63)$ e foi menos elevada na faixa 31 anos ou mais (9,31); a mediana foi igual a 10 na faixa 18 a 20 anos e igual a 9,00 nas outras duas faixas etárias.

\section{Discussão}

Embora muitos autores tenham se dedicado a compreender os motivos que levam as pessoas a praticarem diferentes atividades físicas, este é um dos primeiros estudos que busca identificar os fatores que motivam mulheres adultas a aderir à prática do balé clássico.

A partir dos resultados, pudemos observar que os principais fatores identificados para a busca da prática do balé clássico por mulheres foram o prazer e a sociabilidade. Esses resultados estão em concordância com o que afirmam diversos estudos ${ }^{21,22}$. Ali-Haapala et al. ${ }^{10}$, em um estudo realizado com idosas, identificaram que embora praticar uma atividade física em busca da saúde seja um dos motivos que levam a essa população a praticar o balé clássico, a motivação emocional e a sociabilidade se destacam como os principais fatores.

$\mathrm{Na}$ tentativa de encontrar fatores relacionados à busca pela prática do balé clássico por mulheres acima de 40 anos de idade, Alencastro \& Pinto ${ }^{12}$, fundamen- tando-se em diversos autores, indicam uma série de motivos: busca por uma atividade física visando melhorias na saúde e bem estar físico e emocional através do prazer que uma atividade de dança proporciona; o resgate de lembranças, sensações e sentimentos que acompanham a vida dessas mulheres desde a infância, através da prática na infância ou desejo de fazer balé quando crianças; o estabelecimento de um relacionamento consigo mesmas e com outros sujeitos da mesma escala de interesses ${ }^{12}$.

Um outro estudo que buscou identificar os motivos para a prática de dança, nesse caso a dança de salão, a dimensão prazer foi aquela que apresentou maior resultado entre as participantes para a adesão à prática, indicando que os sujeitos da pesquisa apresentam principalmente o tipo de motivação intrínseca ${ }^{21}$.

A motivação intrínseca também se destacou entre praticantes de balé clássico em estudo realizado com estudantes de diferentes estilos de dança ${ }^{23}$. Quando intrinsecamente motivado, o sujeito participa da atividade por vontade própria, pelo prazer e satisfação do processo de conhecê-la. Comportamentos intrinsecamente motivados, normalmente estão associados com o bem-estar psicológico, interesse, alegria e persistência ${ }^{12}$.

Em nosso estudo foi possível perceber que a motivação para iniciar a prática do balé varia em função da idade, com diferença significativa entre os grupos etários pesquisados para a dimensão "competitividade", demonstrando que as mulheres mais jovens se sentem motivadas a participarem de competições. Embora a

Tabela 4-Estatísticas das dimensões motivacionais segundo a faixa etária

\begin{tabular}{|c|c|c|c|c|}
\hline \multirow{4}{*}{ Dimessão } & \multicolumn{3}{|c|}{ Faixa etária (anos) } & \multirow{4}{*}{ Valor $\mathrm{p}$} \\
\hline & 18 a 20 & 21 a 30 & 31 ou mais & \\
\hline & Média \pm DP & Média \pm DP & Média \pm DP & \\
\hline & Mediana (P25; P75) & Mediana (P25; P75) & Mediana (P25; P75) & \\
\hline \multirow[t]{2}{*}{ Controle de stress } & $26,63 \pm 5,60$ & $24,78 \pm 9,07$ & $30,63 \pm 7,45$ & \multirow[t]{2}{*}{$0,094^{a}$} \\
\hline & $26,50(22,75 ; 29,50)$ & $28,50(18,00 ; 30,50)$ & $30,50(29,00 ; 35,00)$ & \\
\hline \multirow[t]{2}{*}{ Saúde } & $28,13 \pm 9,67$ & $33,33 \pm 7,03$ & $35,44 \pm 6,10$ & \multirow[t]{2}{*}{$0,078^{\mathrm{b}}$} \\
\hline & $27,00(21,00 ; 35,00)$ & $35,50(30,00 ; 37,00)$ & $36,00(29,50 ; 40,00)$ & \\
\hline \multirow[t]{2}{*}{ Sociabilidade } & $37,25 \pm 7,05$ & $38,78 \pm 3,47$ & $38,13 \pm 7,17$ & \multirow[t]{2}{*}{$0,926^{\mathrm{a}}$} \\
\hline & $39,00(31,75 ; 43,00)$ & $39,00(35,75 ; 40,50)$ & $39,50(33,25 ; 45,00)$ & \\
\hline \multirow[t]{2}{*}{ Competitividade } & $16,63 \pm 7,13^{(a)}$ & $11,61 \pm 4,47^{(b)}$ & $9,31 \pm 0,79^{(\mathrm{b})}$ & \multirow[t]{2}{*}{$0,002^{*_{2}}$} \\
\hline & $17,00(10,00 ; 20,00)$ & $9,00(9,00 ; 13,50)$ & $9,00(9,00 ; 9,00)$ & \\
\hline \multirow{2}{*}{ Estética } & $34,75 \pm 6,67$ & $37,33 \pm 5,85$ & $38,19 \pm 4,56$ & \multirow[t]{2}{*}{$0,324^{a}$} \\
\hline & $31,50(30,00 ; 42,75)$ & $38,00(34,25 ; 40,50)$ & $40,00(36,00 ; 40,00)$ & \\
\hline \multirow{2}{*}{ Prazer } & $43,50 \pm 2,78$ & $42,61 \pm 2,89$ & $44,06 \pm 1,91$ & \multirow[t]{2}{*}{$0,212^{\mathrm{a}}$} \\
\hline & $45,00(40,50 ; 45,00)$ & $44,00(40,75 ; 45,00)$ & $45,00(43,50 ; 45,00)$ & \\
\hline
\end{tabular}

* Diferença significativa a 5\%; a = Teste Kruskal Wallis com comparações múltiplas do referido teste; $b=$ teste F (ANOVA) 
dança não se caracterize como uma atividade essencialmente competitiva, e sim artística ${ }^{24}$, é possível observar a existência de diversos festivais e mostras competitivas de dança ao redor do mundo, festivais que funcionam como uma oportunidade entre os jovens de se destacar e muitas vezes conseguir bolsas de estudos em escolas profissionalizantes de grandes companhias de dança $\mathrm{a}^{25}$. A prática do balé buscando a competitividade, em nosso estudo, foi maior entre o menor grupo de idade (18 a 20 anos) e diminuiu de acordo com o aumento da idade, demonstrando que as mais velhas não se sentem motivadas a praticar essa atividade visando competir. Resultado semelhante foi encontrado no estudo de Thesleff ${ }^{23}$, em que se constatou que bailarinos mais velhos se sentem menos motivados a praticar dança a partir da dimensão "competitividade".

Em estudo realizado com adolescentes praticantes de jazz, observou-se que os fatores associados à saúde e competência desportiva, como a competitividade, são mais observados como motivadores a essa prática. Nesses contextos bastante presentes no universo do balé clássico, para os estudantes de dança a prática não se limita à diversão e aprendizagem de coisas novas, mas também sobre ser melhor do que outros e, finalmente, obter um emprego na área ${ }^{23}$. Outros estudos também demonstraram que o fato de ser mais jovem motive mais a prática regular de atividade física também em academias ${ }^{19}$, reafirmando que a competitividade é fator associado à idade no que se refere à prática de diferentes atividades físicas.

À medida que as bailarinas adultas vão ficando mais maduras, é possível observar um sentimento comum, que é o de ter uma prática corporal associada à "poética do dançar", dando um sentido de coletividade ao grupo de praticantes ${ }^{12}$. Por isso, de maneira geral este grupo raramente apresentará traços de competitividade, pois não sente necessidade de alcançar elevados níveis técnicos ou ser solista nos espetáculos; quando a competitividade aparece, é meramente por prazer e descontração, diferentemente daquilo que buscam as mais jovens nessa prática, que são constantemente estimuladas a disputar papeis de destaque ${ }^{12,26}$

Não houve diferenças significativas nas dimensões motivacionais para a prática do balé quando comparados grupos com diferentes tempos de prática ("até 1 ano" e "acima de 1 ano"). Também não foram encontrados estudos que relacionassem os fatores motivacionais para a prática do balé clássico levando em consideração tempo de prática e idade dos participantes.

Um fator limitador da nossa pesquisa diz respei- to ao tamanho da amostra. Sugere-se que este estudo deva ser continuado com uma amostra maior, para determinar com mais clareza os fatores motivacionais para a prática do balé clássico por mulheres adultas. A grande adesão à prática de balé clássico por mulheres adultas ainda é um fenômeno recente e necessita de mais estudos que possam contribuir para sua melhor compreensão. O conhecimento acerca do tema é relevante, principalmente por parte do profissional responsável em orientar e planejar atividades para este público. Essa compreensão poderá possibilitar a realização de um trabalho mais direcionado aos objetivos da população em questão, visando manter a motivação para a adesão e permanência de mulheres na prática do balé clássico, como por exemplo, inscrever as alunas mais jovens em festivais e mostras competitivas e destinar um tempo das aulas para ensaios de coreografias para participação em competições.

É possível concluir que os fatores que motivam a prática do balé clássico por mulheres adultas são diversos, mas de forma geral percebe-se que as mulheres, além de buscar a satisfação pessoal, encontram na prática deste gênero de dança um lugar onde podem firmar laços sociais de amizade. No presente estudo, os principais fatores identificados para a busca da prática do balé clássico por mulheres foram o prazer e a sociabilidade. Conclui-se ainda que a dimensão "competitividade" aparece como um fator motivacional que se destaca entre as participantes mais jovens.

\section{Conflito de interesse}

Os autores declaram não haver conflito de interesse.

\section{Contribuição dos autores}

Oliveira VMM, participou da concepção inicial do estudo, redação e revisão crítica do texto. Lopes JS, foi responsável pela busca da literatura.

\section{Agradecimentos}

Os autores agradecem às escolas de dança envolvidas no estudo.

\section{Referências}

1. Ryan RM, Deci EL. Self-determination theory and the facilitation of intrinsic motivation, social development, and wellbeing. Am Psychol. 2000;55(1):68-78.

2. Martinelli SC, Sisto FF. Motivação de estudantes: um estudo com crianças do ensino fundamental. Aval Psicol. 2010;9(3):413-20.

3. Lopes $\mathrm{P}$, Nunomura M. Motivação para a prática e permanência na ginástica artística de alto nível. Rev Bras Educ Fís Esp. 2007;21(3):177-87. 
4. Paim MCC,Pereira EF.Fatores motivacionais em adolescentes para a prática de jazz. J Phys Educ. 2008;16(1):59-66.

5. Gonçalves MP, Alchieri JC. Motivação à prática de atividades físicas, um estudo com praticantes não-atletas. Psico-USF. 2010;15(4):125-32.

6. Santos SIC, Knijnik JD. Motivos de adesão a prática de atividade física na vida adulta intermediária. Rev Mackenzie Educ Fís Esporte. 2006;5(1):23-34.

7. Marbá RF, Silva GS, Guimarães TB. Dança na promoção da saúde e melhoria da qualidade de vida. RcITPAC. 2016;9(3):8-11.

8. Al Kubaisy W, Mohamad M, Ismail Z; Abdullah NN. Man's and Woman's Motivation to Exercise. AjQoL. 2017;2(8):35-43.

9. 9.Balbinotti MAA, Capozzoli CJ. Motivação à prática regular de atividade física: um estudo exploratório com praticantes em academias de ginástica. Rev Bras Educ Fís Esporte. 2008;22(9):42-44.

10. Ali-Haapala A, Moyle G, Kerr G. Ballet moves for adult creative health: Stage One - Research Report. In: Queensland Ballet [online]; 2018 March; Brisbane. Brisbane: CREATIVE INDUSTRIES; 2018. [citado em 2020 abr 02]. Disponível em: https://eprints.qut.edu.au/117952/.

11. Durante V. Ballet: the definitive illustrated story. New York: DK Publishing, 2018.

12. Alencastro IG, Pinto A. Sensações e motivações: o ballet clássico como prática corporal na idade adulta. In: VIII Encontro de Pesquisa em Arte da FUNDARTE e III Seminário dos grupos de pesquisa da UERGS/Montenegro [online]; 2015 421-24; Bagé. Anais eletrônicos. Bagé: FUNDARTE; 2015. [citado em 2020 mar 25]. Disponível em: file://C:/Users/vivim/Downloads/298-428-1-PB.pdf.

13. Caminada E, Aragão V. Programa de ensino de ballet: uma proposição. Rio de Janeiro: Comunicação Corporal, 2013.

14. Whitehill A, Noble W. The parents book of ballet: answers to critical questions about the care and development of the young dancer. 2nd edition. Colorado: Meriwether Publishing Ltd., 2003.

15. Moller A, Masharawi Y. The effect of first ballet classes in the community on various postural parameters in young girls. Phys Ther Sport. 2011;12(4):188-93.
16. Barringer J, Schlesinger S. The Pointe Book: Shoes, Training \& Technique. Hightstown, NJ: Princeton Book Company, 2004.

17. Dumith SC, Domingues MR, Gigante DP. Estágios de mudança de comportamento para a prática de atividade física: uma revisão da literatura. Rev. Bras Cineantropom Desempenho Hum. 2008;10(3):301-07.

18. Balbinotti M. Inventário de motivação aplicado a atividade física. Porto Alegre: Laboratório de Psicologia do Esporte/ Universidade Federal do Rio Grande do Sul, 2003.

19. Freitas CMSM, Santiago MS, Viana AT, Leão AC, Freyre C. Aspectos motivacionais que influenciam a adesão e manutenção de idosos a programas de exercícios físicos. Rev Bras Cineantropom Desempenho Hum. 2007;9(1):92-100.

20. Junior JO, Guariglia D, Loch M. Adesão e aderência a um programa de exercício físico em pessoas vivendo com HIV/ AIDS. Rev Bras Ativ Fís Saúde. 2017;22(6):568-75

21. D’Aquino R, Guimarães ACA, Simas, JPN. Dança de Salão: Motivos dos indivíduos que procuram esta atividade. Efdeportes. 2005;10(88):17-23.

22. Abreu EV, Pereira LTZ, Kessler EJ. Timidez e motivação em indivíduos praticantes de dança de salão. Conexões. 2008;6(1):649-64.

23. Thesleff, P. Participation in Dance Training in Finland- A Study of Motives and Behavior Regulation. [Unpublished Master's Thesis in Sport and Exercise Psychology]. Yväskylän yliopisto, Finlândia: University of Jyväskylä. Department of Sport Sciences; 2014.

24. Marques I. Ensino da dança hoje: textos e contextos. São Paulo: Cortez, 1999.

25. Wyon M. Preparing to perform: periodization and dance. J Dance Med Sci. 2010;14(1):67-72.

26. Lira CB. O que aprendi com a sapatilha de ponta: um olhar feminista de uma bailarina quase balzaca. Salvador: Programa de Pós-Graduação em Estudos Interdisciplinares sobre Mulheres - UFBA, 2017.

Recebido: $17 / 05 / 2020$

Aprovado: 18/08/2020

\section{Como citar este artigo:}

Oliveira VMM, Lopes JS. Motivação para a prática de balé clássico por mulheres adultas. Rev Bras Ativ Fís Saúde. 2020;25:e0129. DOI: $10.12820 /$ rbafs.25e0129 